\title{
Practical Manual on Veterinary Clinical Diagnostic Approach
}

\author{
Ararsa Duguma* \\ Haramaya University, College of Veterinary Medicine, Ethiopia
}

\begin{abstract}
Veterinary clinical examination relies on knowledge of Anatomy, Physiology, Pathology and Animal behavior, skills in the methods and techniques of clinical examination, clinical sign and pathogenesis of the diseases which are the basic requirements for clinician in his/her good diagnostic approach. Disease problems in veterinary medicine are invariably presented to the clinician through the medium of the owner's complaint, which is a request for professional assistance by giving animal history. In any clinical examination procedures, it is necessary to employ some suitable means of restraint: physical, chemical or verbal, in order to be able to carry out the examination safely and without danger to the clinician or his assistants. Inspection, Palpation, percussion and auscultation are the commonly used methods of physical examination for assessing pathophysiological or anatomical abnormalities of given animal during clinical examination. The general clinical examination involves detailed consideration of physical body condition; conformation/shape; posture; gait; abnormal behavior; body temperature; pulse; and respiration of individual animal; whereas the regional or systematic clinical examination involves the application of the various clinical methods to the various regions or systems of the body. The system involved is identified and is then examined in greater detail using either a complete or a problem oriented examination. In addition to history taking and clinical examination; skills on techniques of laboratory sample collection and submission is an important consideration for further diagnosis, treatment and take practicable control and prevention measures on identified disease.
\end{abstract}

Keywords: Clinical examination; History taking; Physical method; Restraint; Sampling techniques

\section{Introduction}

In the investigation of any animal disease problem, the veterinarian must, of necessity, undertake a careful and thorough clinical examination with the object of recognizing the nature of the affection, so that effective treatment and, where practicable, control measures are adopted. The situation is rendered complex by the necessity to deal with a variety of species of domestic animals and birds. Increasing specialization on the part of practicing veterinarians will resolve some of the apparent problems thus presented. In general, however, the same principles may be applied in all cases to deal with the diverse difficulties that clinical diagnosis presents.

The organs or systems involved, the location, type of lesion present, the pathophysiological processes occurring and the severity of the disease can be deduced from the information gained during the clinical examination. Without a proficient clinical examination and an accurate diagnosis it is unlikely that the control, prognosis and welfare of animals will be optimized.

The success of clinical examination relies heavily on the knowledge of the clinician and usually assumes a single condition is responsible for the abnormalities. Many clinicians begin their examination by performing a general examination which includes a broad search for abnormalities [1,2]. The system or region involved is identified and is then examined in greater detail using either a complete or a problem oriented examination. For this sound knowledge of Anatomy, Physiology, Pathology and Animal behavior, skills in the methods and techniques of clinical examination, knowledge of etiology, clinical sign and pathogenesis of the diseases are the basic requirements for clinician to make diagnosis. This manual is a practical guideline for veterinary practitioner with the following objectives:

- Guide to apply methods of animal handling/restraint and ability to take history

- A guide for general and systemic physical clinical examination for further diagnosis, treatment and take practicable control and prevention measures on identified disease

- Having knowledge on preparation and administration of veterinary drugs

- Gives skills on veterinary laboratory sampling techniques

\section{History Taking}

Disease problems in veterinary medicine are invariably presented to the clinician through the medium of the owner's complaint, which is a request for professional assistance. For completeness and accuracy of history taking, the following points should be well considered (Patient data, Immediate/present history, past history, Management and Environment history).

\section{Patient data}

This data is essential for accurate identification of the patient and includes:

- Owner's name

- Owner's address: postal address, telephone, kebele, peasant association, etc.

- Species, breed, sex, age, name, ID No., body weight, etc.

- Description including color, marking, polledness, and other identification marks of patient

*Corresponding author: Ararsa Duguma, Haramaya University, College of Veterinary Medicine, College of Veterinary Medicine, PO Box 138 Dire Dawa, Ethiopia, Tel: +251926260484; E-mail: ararsad@yahoo.com

Received March 16, 2016; Accepted May 19, 2016; Published May 23, 2016

Citation: Duguma A (2016) Practical Manual on Veterinary Clinical Diagnostic Approach. J Vet Sci Technol 7: 337. doi:10.4172/2157-7579.1000337

Copyright: @ 2016 Duguma A. This is an open-access article distributed under the terms of the Creative Commons Attribution License, which permits unrestricted use, distribution, and reproduction in any medium, provided the original author and source are credited. 


\section{Present history}

This relates to the sequence of events associated with the period of time that the animal has been ill. Points to be focus in present history are:

- Duration of the disease: whether it is peracute, acute, subacute or chronic

- Clinical sign/symptoms: (appetite for food or drink, defecation, urination, respiration, sweating, physical activity, milk production, growth, gait, posture, voice, odour, etc.)

- The number of animals affected: (morbidity rate and mortality rate)

- Treatment given: determine whether any treatment has, in fact, been given before calling for assistance

\section{Past history}

In this respect, information should be obtained relating to the nature and timing of any previous illness which had affected the individual animal or group.

- Details regarding clinical features, diagnosis, treatments, morbidity and mortality rates, post mortem observations, laboratory test etc., should be obtained [3].

- Ascertain the system of animal replacement on the farm or in the home.

- If animal introduced from outside sources, further enquiries should be made concerning the health history and status of the source animals.

\section{Management and environmental history}

The examination of an animal must be accompanied by a consideration of its surroundings and circumstances.

\section{Management:}

- Nutrition,

- Livestock at pasture,

- Drinking water,

- Feeding methods/practice

- House space, satisfactory ventilation

- Proper management of milking cow and milking machine to avoid udder injury

- Breeding and Reproductive history

- Stocking rate/population density

Examination of the environment:

- Topography

- Soil type

- Ground surfaces

- Climatic conditions

- Environmental Hygiene

- An excessive buildup of feces and urine

- Quality of Floor

\section{Methods of Restraint}

Since animals often resist many of the clinical examination procedures, it may be necessary to employ some suitable means of restraint, in order to be able to carry out the examination safely and without danger to the clinician or his assistants. The methods available may be classified as:

- Physical restraint when various instruments are employed.

- Chemical restraint when drugs inducing varying degrees of sedation or immobilization are administered.

- Verbal/Moral restraining which can be more practiced by owner.

\section{Physical restraint}

It is important to perform all the physical manipulations in a quiet and gentle manner in order to carry out the examination safely without causing danger to the clinician or his assistants and to avoid disturbing the patient.

Restraining methods for equine, cattle, Pet animal, sheep and goats.

Training materials: open air clinic

Live animals

Rope, nose twitch, bull holder, crash etc.

\section{Procedure:}

\section{Restraints of the equine:}

- Twitch is applied to the upper or lower lip or to the ear

- Nose twitch

- Lifting the fore-leg and hind-leg by unaided hands or with Leg twitch

- A loop of strong cord or soft rope is applied to the appropriate part

- Two ropes one-person horse casting

- Two ropes four persons horse casting

\section{Restraint of the cattle:}

- The nasal septum is gripped between the thumb and one finger or with 'bull-holder

- Leg twitches are also employed

- One rope locking two horns on a post or tree

- One rope two person cattle casting

- Two ropes three person cattle casting

Restraint of sheep and goat:

- One person holds the neck of the sheep or goat by two hands

- One person stands beside the sheep or goat embracing the animal

- Small animals are restrained by placing them on a table in the upright, lateral or dorsal position

Pet animals

- Placing them on a table in the upright, lateral or dorsal position 
- In the dog a tape muzzle or a leather muzzle is used

\section{Chemical restraint}

Drugs that is useful for this purpose includes:

- Acepromazine, Acetylpromazine, Chlorpromazine, Promazine and Trimeprazine; members of this group can be used in most species of animals.

\section{Verbal/moral restraining}

It is more practiced by owner e.g., feed provision, massaging, calling name of animal etc.

\section{Physical examination methods}

\section{Objective}

To apply general inspection, palpation, percussion and auscultation methods used to detect clinical signs of abnormalities.

\section{Training materials}

- Live animals (equine, cattle, sheep and goats)

- Pleximeter, hammer

- Stethoscope

- Gloves

\section{Procedure}

General inspection: It is done some distance away from the animal; sometimes go round the animal or herd/flock, in order to get the general impression about the case [4].

- Attention should be paid to the following items: (Behavior, Appetite, Defecation, Urination, Pasture, Gait, Body condition, Body conformation)

- Lesions on outer surface of the body can be observed: (Skin and coat, Nose, Mouth, Eyes, Legs and hoofs, Anus)

\section{Palpation:}

Objective: To detect the presence of pain in a tissue by noting increased sensitivity

Method: Use fingers, palm, back of the hand, and fist, in order to get the information about the variation in size, shape, consistency and temperature of body parts and lesions, e.g., the superficial lymph nodes.

The terms, which can be used to describe the consistency of parts during palpation, are:

- Resilient, when a structure quickly resumes its normal shape after the application of pressure has ceased (e.g., Normal rumen)

- Doughy, when pressure causes pitting as in edema

- Firm, when the resistance to pressure is similar to that of the normal liver (e.g., neoplasia/tumor)

- Hard, when the structure possesses bone-like consistency (e.g., Actinomycotic lesion)

- Fluctuating, when a wave-like movement is produced in a structure by the application of alternate pressure (e.g., hernia, hemorrhage/hematoma)
- Emphysematous, when the structure is swollen and yields on pressure with the production of a crepitating or crackling sound (e.g., Black leg) [5].

\section{Percussion:}

Objective: To obtain information about the condition of the surrounding tissues and, more particularly, the deeper lying parts. Percussion can examine the area of the subcutaneous emphysema, lungs, rumen and rump

Method: By means of striking a part of the body to be percussed

Immediate percussion: Using fingers or hammer directly strike the parts being examined.

Mediate percussion: Finger-finger percussion; Pleximeter-hammer percussion

The quality of the sounds produced by percussion is classified as:

Resonant: which is characteristic of the sound emitted by air containing organs, such as the lungs.

Tympanic: The sound produced by striking a hollow organ containing gas under pressure, e.g., tympanitic rumen or caecum.

Dull: Sound emitted by a solid organ like the liver or heart [6].

\section{Modified percussion:}

- Ballottement percussion: Used to detect late pregnancy in small ruminants, dogs and cats

Procedure: Apply a firm and interrupted push on the uterine region of the abdomen of small ruminants

Detection of rebound of floated material shows pregnancy

- $\quad$ Fluid percussion: Used to detect fluid in the abdomen

Procedure: Apply a push on one side of the abdomen, percussion on the other side

The presence of wave-like fluid movement shows accumulation of fluid in the abdomen, e.g., ascites [7].

\section{Auscultation:}

Objective: To listen the sounds produced by the functional activity of an organ located within a part of the body. This method used to examine the lung, trachea, heart and certain parts of the alimentary tract.

\section{Direct auscultation:}

Procedure: Spread a piece of cloth on the part to be examined using two hands to fix the cloth and keep your ears close to the body, then listen directly.

Indirect auscultation: Use stethoscope.

Procedure: Fix the probe of the stethoscope firmly on the part of the body to be examined and listen to the sounds produced by the functional activities of the body carefully.

\section{Clinical Examination of the Patient}

\section{General physical examination}

\section{Temperature taking:}

Materials:

- Live animals 
- Thermometer

- Lubricant (soap or petroleum jelly), Antiseptics

\section{Procedure:}

- The places, which can be used to take temperature are rectum or vagina (approximately 0.5 degree centigrade higher in vagina).

- The thermometer should be sterilized by disinfectant (antiseptics) before use.

- It should be well shaken before recording of temperature to bring the mercury column below the lowest point likely to be observed in different species of animals.

- The bulb end of the thermometer should be lubricated with liquid paraffin or glycerin or soap especially in case of small pup and kitten.

- Insert the thermometer in a rotational way and gentle manner. Care should be taken so that the bulb of the thermometer remains in contact with the rectal mucous membrane.

- The thermometer should be kept in site for at least 3-5 minutes.

- Pull out the thermometer, clean it and read the number.

- Evaluation: Read the value to define and explain a state of fever, hypothermia, and febrile or non-febrile animals [8].

Pulse taking: Pulse can be adapted from the number of heart beats per minute by using stethoscope in less manageable animals. The rhythm of pulse should also be noticed while taking pulse.

\section{Procedure:}

- Place the digits on the artery to be examined

- Applying gentle pressure until the pulse wave can be detected

- Note the pressure or pulsation of the arteries felt on the finger digits

- Count the number of beats per minute (counting should be done at least for 30 seconds and multiplied by 2); notice the quality and rhythm of pulse (Table 1).

\section{Materials}

- Live animals

- Stethoscope

- Watch

\section{Respiration taking}

Materials:

- Live animals

\begin{tabular}{|c|c|c|}
\hline S No & Species of animal & Site of pulse taking \\
\hline 1 & Horse & $\begin{array}{c}\text { External maxillary artery } \\
\text { Transverse facial artery } \\
\text { Median artery } \\
\text { Great metatarsal artery }\end{array}$ \\
\hline 2 & Cattle & $\begin{array}{c}\text { Facial artery } \\
\text { Median artery } \\
\text { Middle coccygeal artery }\end{array}$ \\
\hline 3 & Sheep/goat, dog, cat, pig, and calf & Femoral artery \\
\hline
\end{tabular}

Table 1: Site of pulse taking for different species of animals.
- Stethoscope

- Watch

- Gloves

- Crash

Method: The respiration rate is measured through counting of either contraction or expansion of the thorax and abdomen which can be observed during clinical examination

Procedure: A method for respiration rate taking includes:

- Inspection: Stand behind and to one side of the animal, and observe the movement of the thoracic and abdominal areas of the body.

- Palpation: Put one hand in front of the nostril, feel the exchange of the gas; or put one hand on the lung area or the thorax and feel the respiratory movements.

- Auscultation: Use stethoscope, listen to the respiration sound in the trachea or lung area.

\section{Capillary Refill Time (CRT):}

\section{Method:}

- This is taken by compressing the mucosa of the mouth or vulva to expel capillary blood, leaving a pale area

- Recording how long it takes for the normal pink color to return.

- In healthy animals the CRT should be less than 2 seconds.

- A CRT of more than 5 seconds is abnormal, and between 2 and 5 seconds may indicate a developing problem

Physical body condition: Body condition scoring is an important management practice used by producers as a tool to help optimize production, evaluate health, and assess nutritional status. Different scores can be given for individual animal and can further classified as normal, fatty, lean/thin, emaciation.

Condition Score 1: Very thin: This animal's skeletal structure is very prominent. Notice the deep depressions next to the spine, between the pelvis and rib cage, between the hooks and pins, and around the tail head.

Condition Score 2: Thin: The animal's skeleton is still very apparent. The individual spinous processes are clearly visible, but there is a small amount of fat tissue over the spine, hooks, and pins.

Condition Score 3: Medium (Normal body condition): The animal appears smooth over the spine, ribs, and pelvis and the skeletal structure can be easily palpated. The hooks and pins are still discernible, with a moderate, rather deep depression between the pelvis and rib cage, hooks and pins, and around the tail-head.

Condition Score 4: Fat: There are no spinous processes detectable, and no depression in the loin area, which gives the top-line of the animal a flat, tabletop appearance. The ribs can no longer be felt, and the pelvis can only be felt with firm pressure. The hooks and pins have a rounded appearance due to areas of fat covering.

Condition Score 5: Very Fat: The animal appears rounded and smooth with a square-shaped appearance, because of the amount of fat filling in the loin. The skeletal structure is no longer visible, and can only be palpated with excessive pressure 
Normal demeanor: When, on being approached, an animal makes a normal response to external stimuli, such as movement and sound, the demeanor is said normal (bright). Normal reaction under these circumstances may consist of elevating the head and ears, turning towards and directing the attention at the source of stimuli, walking away and evincing signs of attack or flight.

Abnormal demeanor: Behavioral change/ response to external stimuli.

- $\quad$ Decreased response (depression): dull (apathetic); dummy state; comma.

- Excitation or increased response: apprehension (mildly anxious); restlessness; mania; frenzy.

- Posture: It denotes the anatomical configuration when they remain in stationary situation. How does it stand? How does it sit? How does it lie?

- Gait: It indicates about the locomotory process of an animal.

- Body conformation: shape and size of the different body regions relative to other regions

\section{Regional or systematic clinical examination}

Clinical Examinations of the head and neck region:

Objective: To identify pale and discolored mucus membranes; assess problems of oral cavity and deranged appetite. The following points are to be considered:

- Visible mucous membrane

- $\quad$ Eyelids, conjunctivae and eyes

- Nasal regions and nasal mucous membrane

- Prehension, mastication and deglutition

- Salivation

- Teeth eruption

Materials: Live animals, crash and glove

\section{Procedure:}

- Visible mucous membrane examined by visual inspection to note the presence of lesions, discharge, glaucoma, nystagmus.

- Examine the nose and nasal sinuses; lesions, discharges should be noted by percussion, palpation

- Examine the mouth and appetite; oral lesions, salivation, feed intake should be noted

Examination of skin and appendages: Structures or parts associated with skin as its appendages are hoofs, hairs, horns, quills, claws, nails, sebaceous glands and sweat glands.

Objective: Assessing the condition of skin and coat to identify clinical signs of skin lesions such as:

- Condition of the coat

- Elasticity of the coat

- $\quad$ Pruritus

- $\quad$ Primary and secondary skin lesions

- Dermatitis
- Hyperkeratosis or parakeratosis

- $\quad$ Presence of ectoparasites

Materials: Live animals, crash, glove

\section{Procedure}

Examine the skin and coat: grasp the skin of the upper part of the body and notice the elasticity, visual inspection of the condition of the coat and presence of skin lesions should be noted.

\section{Examinations of the thoracic cavity:}

Objective: to show the regional anatomy of the lungs and the heart, and perform physical examination of the lung and the heart area

\section{Materials:}

- Live animals

- Pleximetre and percussion hammer

- Stethoscope

\section{Procedure:}

Regional anatomy of the lungs -locate the lung area

The lung is located on the external surface of the thoracic region by forming an imaginary triangle by using the points at the angle of the scapula, olicranun process and the second intercostals space from the last.

\section{Physical examination of the thorax (lung area)}

- Inspection -note respiratory movements

- Palpation -check the presence of pain by applying pressure

- Percussion -notice resonant sound

- Auscultation -note bronchial sounds (trachea and anterior part of the lungs) and alveolar sounds (Figure 1)

\section{Regional anatomy of the heart -locate the heart area}

The heart is suspended by great vessels and located on the left median mediastinum of ventral thorax. The left side of the heart apex reaches the chest wall.

- In horse, heart is located between $2^{\text {nd }}$ to $6^{\text {th }}$ intercostals space

- In cattle, $3^{\text {rd }}$ to $6^{\text {th }}$

- $\quad$ In camel, $3^{\text {rd }}$ to $7^{\text {th }}$.

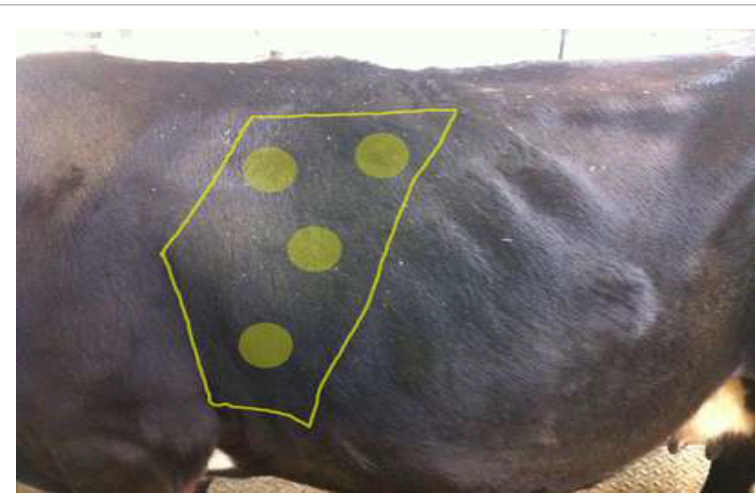

Figure 1: Anatomical location of lung in cow. 


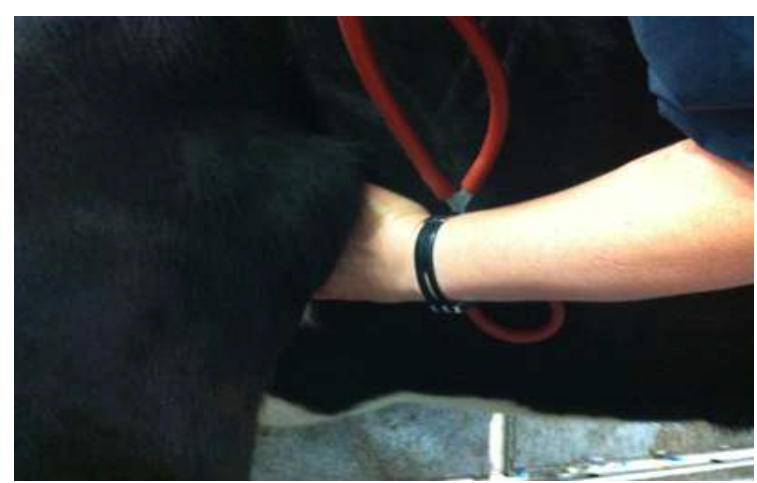

Figure 2: Auscultation to assess heart sound in cow.

After locating the heart the following should be noted through physical examination (palpation, percussion, auscultation)

- Heart rate

- Abnormal variation in heart rate

- Heart sounds

- Normal heart sounds (dub-lab)

- $\quad$ Adventitious heart sounds (murmurs)

- $\quad$ Pericardial frictional sounds

- Venous pulsation (jugular pulsation) (Figure 2)

Clinical examinations of the abdominal and associated digestive organs

Objective: to undertake clinical examination of the abdomen and identify disturbances of the digestive system.

\section{Materials:}

- $\quad$ Live animals

- $\quad$ Stomach tube

- $\quad$ Gloves

- Lubricant

- Trocar and cannula

Procedure: Applied anatomy of the abdominal region of animal

General clinical examination of the abdominal region

Clinical examination of the stomach and intestines

- The examination of rumen is performed by inspection, palpation, percussion and auscultation, stomach tube can be used as well. In bloat case, the left side would be bulged, and the motility would be decreased.

- The examination of reticulum: TRP by back grasp, below pole lifting, up and down hill leading, hand palpation

- The examination of omasum: done by exploratory puncture.

The examination of the displacement of abomasums is done by inspection, palpation, and auscultation.

The disorder of stomach can be detected by taking stomach contents sample: Insert the stomach tube or nasal tube after cleaning, disinfecting and lubrication or trocarisation (Figures 3 and 4)

- Rectal examination of the internal abdominal structures: cut and smooth the nail; wear shoulder long glove; lubricate; cone shape of the fingers; insert in rotating way; notice: the hand cannot open, or even grasp organs inside. It's necessary or possible to use tranquilizer to reduce the sensitivity of the rectum in horse. In bloat case, the pressure in the abdomen would be very high, so it would be difficult to insert the hand inside (Figure 5).

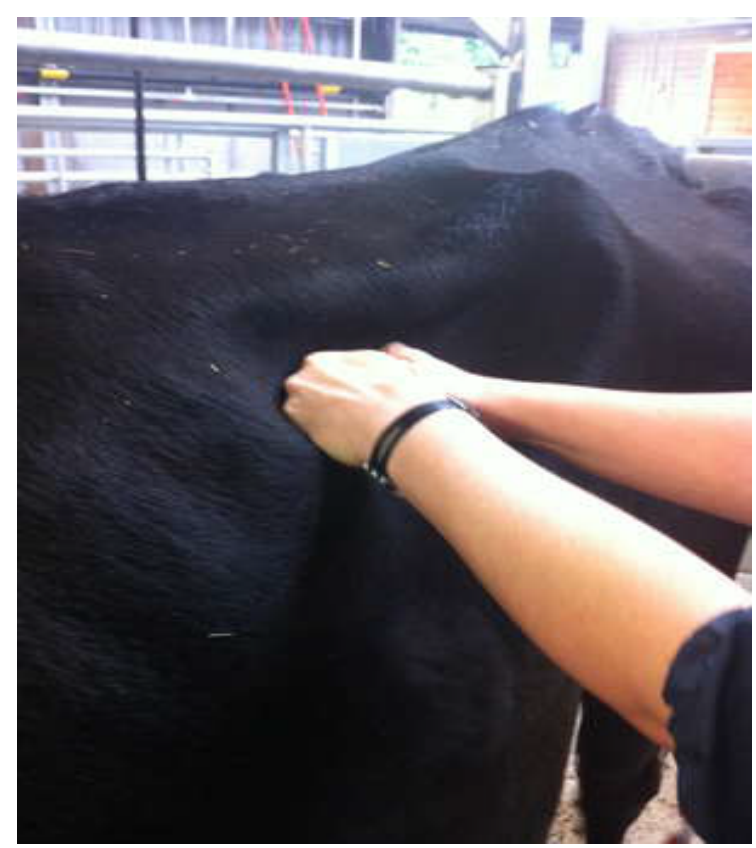

Figure 3: Feeling rumen contraction by palpation.

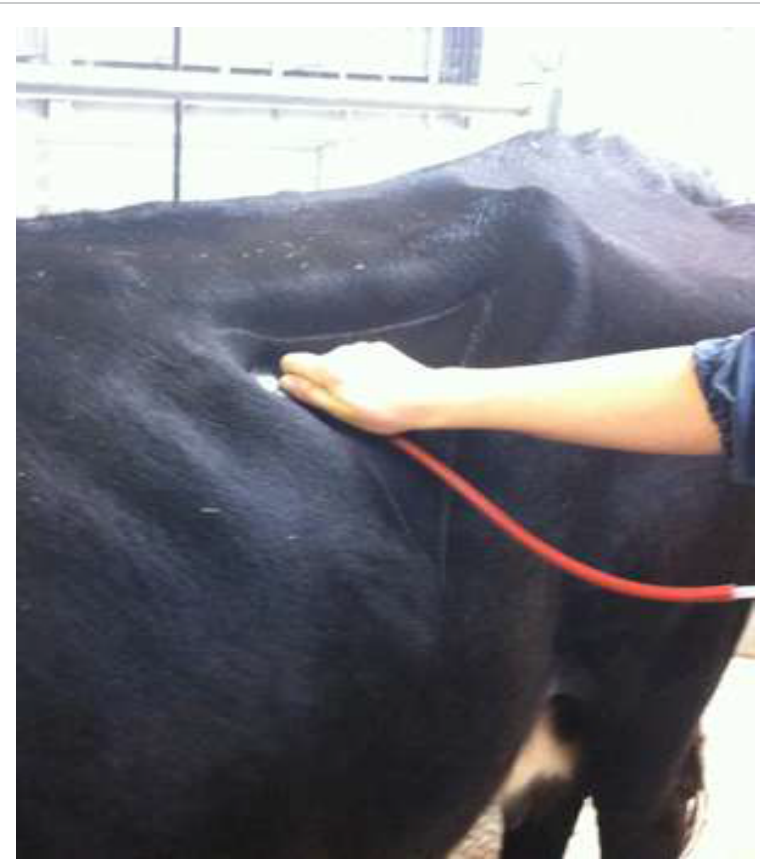

Figure 4: Auscultation of rumen contraction. 


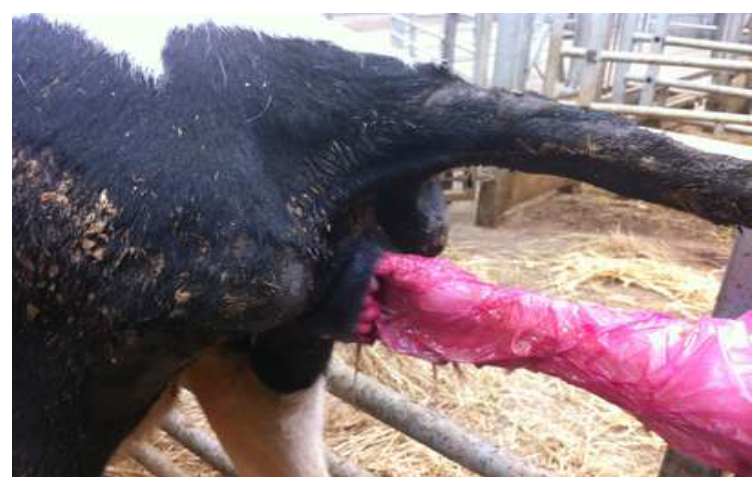

Figure 5: Rectal examination of cow.

\section{- $\quad$ Clinical Examinations of the Feces}

Objective: To see Character of the feces and abnormal constituents in feces

\section{Procedure}

- Observe the surface of the faeces, where mucus and blood always exists

- The colour of the faeces surface, the odour of the feces

- Fishy smell generally indicates bleeding, and abnormal constituents in feces

\section{Examination of the urogenital system:}

Objective: To identify the regional anatomy, undertake clinical and physical examination of urinary system and assess urinary abnormalities, perform clinical examination of female and male reproductive organs as well as the mammary glands and teats.

\section{Materials:}

- $\quad$ Live animals, milk

- Catheter

- Speculum and artificial light

- $0.1 \%$ potassium permanganate

- lubricant

\section{Procedure:}

Identify the anatomic structure of the kidney on live animal: the left kidney is located ventral of the transverse process of the $3^{\text {rd }}$ to $5^{\text {th }}$ lumbar vertebrae. The kidney can be examined by inspection, external palpation, rectal palpation, and urianalysis.

- The examination of the urinary bladder is performed by stimulating the sense of urination from the lower part between the two hind legs on small animals or rectal palpation on large animals. Attention should be paid to the paralysis of the bladder and retention of urine and rupture.

- Clinical examination of male genital organs- visual inspection and palpation are employed to examine the testes, prepuce and the penis after withdrawing from the prepuce.

- Here orchitis, cryptorchidism, scrotum hernia, obstruction of urethra by calculi, phimosis, paraphimosis, inflammation of the prepuce, testes and penis are noted.
- Clinical examination of female genital organs- visual inspection and palpation of the vaginal region, use of vaginal speculum to examine the inside of vagina and intrarectal examination of the cervix, uterus and ovaries would be performed

- Take the sample of urine examine through physical methods

- Fresh urine is collected with test tube after stimulating urination by palpating the perennial region or by inserting catheter

- Note how to insert catheter.

- Clinical examination of the mammary glands and teat- inspection and palpation to detect the presence of swelling and lesions on the teats/decrease in size and shape, any discharge, temperature of the udder, consistency, and pain reactions are performed

- Clinical examination of milk samples: after collecting the milk samples in clean test tubes one can apply different physical and laboratory examinations

- For the gross examination of the milk, the change of the color, odor, viscosity and flakes in the milk should be identified.

\section{Examinations of the nervous and musculoskeletal system:}

Objective: For examination and detection of clinical signs of various problems associated with the nervous and musculoskeletal systems

Materials: Live animals, Audiovisuals and pictures

\section{Procedure:}

The examination of the nervous system:

1. Observe the behavior of the animal

2. Note responses of the animal while applying different stimuli

- Examine the brain by corneal reflex, the pupil reflex

- Examine the spinal cord of the neck and thorax by withers reflex

- Examine the spinal cord of the back part by applying hoof stimuli and anus stimuli

- Move the animal to and from or uncomfortable position to examine the locomotors

3. Examine the joints long bones and different muscles: arthritis, dislocation of the joints, rickets or osteomalacia, muscular atrophy, central or peripheral origin paralysis

\section{Examination of superficial lymphnodes of animals}

Objectives: to compared each paired node for size and consistency with the contralateral node.

\section{Method:}

Grossly enlarged lymph nodes may have been seen during observation of the patient before it is handled. Observation and palpation is possible when the animal is restrained.

\section{Anatomical Location of lymphnodes (LN):}

- Submandibular lymph nodes: situated and are palpable on the medial aspect of the 'angle of the jaw' where the horizontal and vertical rami of the mandible meet. (Normal size is 1.5 to $2 \mathrm{~cm}$ )

- Prescapular lymph nodes: It lies subcutaneously and underneath the cutaneous muscle just anterior to the shoulder joint (Normal size $1 \mathrm{~cm} \times 3.5 \mathrm{~cm}$ ) 
- Axillary lymph nodes: Found on each side of the chest in the armpit area. Normally only palpable in young calves without heavy muscling (Normal size $1.5 \mathrm{~cm}$ )

- Popliteal lymph nodes: These nodes are found surrounded by dense muscle tissue immediately behind the stifle. It found on each rear leg on the opposite side of the knee. They are relatively superficial and easy to feel (normal size 1-1.5 cm)

- Inguinal lymph nodes: These are usually palpable as a small group of fairly mobile and firm structures adjacent to the inguinal canal.(normal size $0.5 \mathrm{~cm}$ )

- Supramammary lymph nodes: These are normally readily palpated on the caudal aspect of the udder just above the upper limit of the mammary glandular tissue. (normal size $2.5 \mathrm{~cm}$ ) (Figure 6)

\section{Preparation and Administration of Medicaments}

\section{Drug dosage form}

Oral dosage forms: Refers to administration of drug through the mouth. The most commonly used preparations are solid oral dosage forms such as tablets, capsules, granules, powder, paste and boluses etc.

Parentral dosage forms: the most common parentral dosage forms are stable aqueous solutions and subcutaneous implants.

\section{External dosage forms:}

- Ointment- semisolid preparation for external application.

- Cream- a viscous semisolid, consisting of oil in water emulsion or water in oil emulsion.

- Dusting powder e.g., popular antibacterial agent applied on animal wounds.

- Lotion- an aqueous solution or suspension for local application.
- Spray-a drug applied in liquid form by pressure.

Inhalation dosage forms: gaseous and volatile liquid anaesthetic agent (drugs), given by inhalation, e.g., Halothane

\section{Routes of drug administration}

Oral administration: There are large numbers of pharmaceutical preparations available for oral administration. Solid dosage forms (powders, tablet, capsules, pills, etc.) and liquid dosage forms (syrups, emulsion, mixture, drench, electrolytes, etc.)

Parenteral administration (IV, IM, SC, Id, epidural, subconjunctival): It refers to a drug administration by injection directly in to the tissue fluid or blood without having to cross the intestinal mucosa.

Intravenous route (IV): Gives swift, effective and highly predictable blood concentration and allows rapid modification of dose and is used for emergency treatment. In most animals (horse, cattle, sheep and goat) usually given through jugular vein, in pig-ear veins, in the dog and cat-cephalic vein and recurrent tarsal vein.

Intramuscular (IM) route: Absorption occurs either haematogenous or via lymphatic and is usually fairly rapid except for long acting preparation.

Subcutaneous (SC) route: Preferred when slow and continuous absorption of drug is required. The injected drug disperses through the loose connective tissues. They dissolve in tissue fluid before it can enter either capillaries or lymphatic.

Intradernal route (ID): Used for testing hypersensitivity test and for vaccination.

Epidural route: Refers to deposition of drug up on or outside the dura matter.

E.g., Introduction of local anaesthetics between the first and second coccygeal vertebra to eliminate straining.

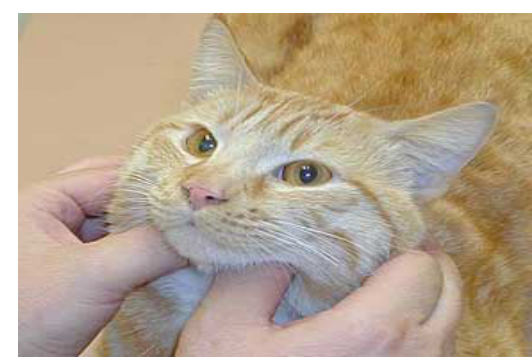

Submandibular LN

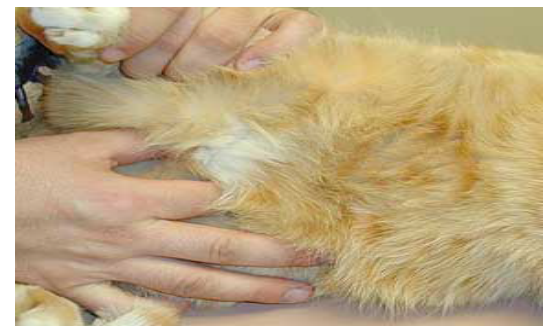

Inguinal LN

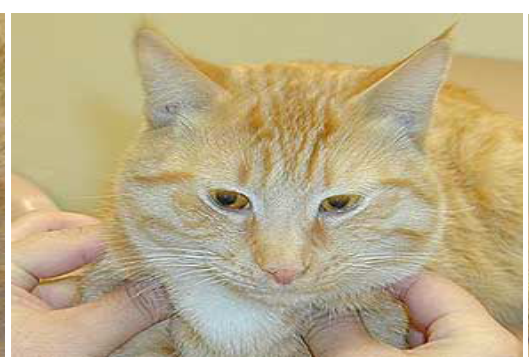

Prescapular LN

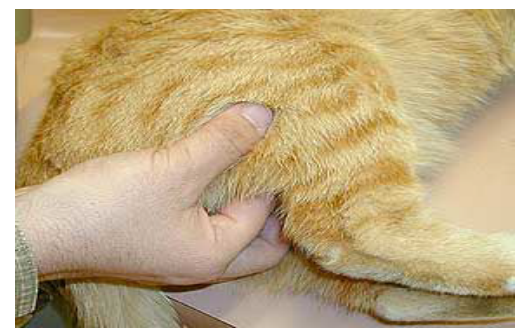

Popliteal LN

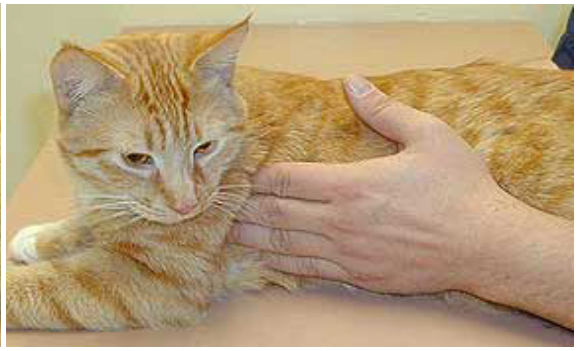

Axillary LN

Figure 6: Anatomical location of superficial lymph node. 
Subconjunctival: Disposition of a pharmaceutical preparation beneath the conjunctiva.

Topical or local application: It refers to external application of drug to the body surface for localized action at accessible site, such as skin, eyes, body orifices, body cavity.

\section{Drug dose calculation}

Dose is the quantity of the drug to be administered at one time and expressed in $\mathrm{mg} / \mathrm{kg}$ or IU $/ \mathrm{kg}$.

$$
\text { Dose }: \frac{\text { Body weight } \times \text { Dose Rate }}{\text { Concentration }}
$$

E.g., A $5 \mathrm{~kg}$ cat needs an antibiotic at a dose of $15 \mathrm{mg} / \mathrm{kg}$. The antibiotic comes in liquid form at concentration of $25 \mathrm{mg} / \mathrm{ml}$ how many $\mathrm{ml}$ do you give?

Solution:

$$
\text { Dose }: \frac{5 \mathrm{~kg} \times 15 \mathrm{mg} / \mathrm{kg}}{25 \mathrm{mg} / \mathrm{ml}}=3 \mathrm{ml}
$$

\section{Veterinary Laboratory Sampling Techniques}

\section{Sample submission requirements} details.

Ensure all information is completed and contains all relevant case

\section{Labeling of specimens:}

- Type specimen (e.g., Serum, whole blood, liver, brain, urine, etc.), whether preservative is used or not and type of preservative used.

- An identification of animals (e.g., tag, name, colors of hair coat, etc.) if specimens were collected from more than one animal.

- The names of the owner, if specimens from different cases are packed together in the same parcel.

- Date of collection.

\section{Handling and transport of specimens}

- The parcels should be securely packed in order to withstand rough handling and protect shipper and the environment

- Great care should be taken to ensure that the containers with specimens do not leak! (a sticky tape may be used)

- Use cotton wool, newspaper or other absorbent materials to soak up any leakage that, despite all the precautions, might occur.

- Small cooler (Styrofoam) boxes are preferred for shipping refrigerated specimens.

\section{Collection of specimen}

Whole blood:

- Aseptically bleed directly in to vacutainers containing EDTA or heparin preservatives

- Mix gently by rolling the tube

- Do not freeze but keep cool on ice

Serum:

- Use red topped vacutainer tubes for serology and mineral analysis
- After collecting the blood sample, the tube is left untouched at room temperature till clotting is complete

- The clot is then carefully removed and then Centrifuge or allow any remaining cells to settle

- Gently pour the clear serum into a fresh or sterile tubes or cryovials

- Care must be taken to keep the serum completely free from blood cells

- Keep refrigerated (or $-20^{\circ} \mathrm{C}$ ) if the serum stay longer at your laboratory/clinic before shipment

\section{Blood smears:}

- Remember to shake the blood gently while collecting blood to prevent clotting.

- Use clean, dry smears

- Take a small drop blood and place on right end of one slide about $1 \mathrm{~cm}$ from edge

- Place another glass slide (spreader) to the left of drop at $30^{\circ}$ angle gently touching the drop

- Wait until blood has spread all way across the glass (spreader) slide

- Move spreader slide to the left quickly but smoothly

- Dry by waving in the air

Note:

- Use dry, non-greasy glass slides.

- Must be dried before wrapping in clean tissue paper.

- Protect from rain, flies, dust and abrasion.

- Submit at least 6 slides for a single case.

- Label smears clearly with pencil

- Pack individually.

Faeces:

- Should be fresh and free of soil and vegetation

- Collect direct from rectum

- Lubricate your finger after wearing glove and rotate for easy entrance without damage the mucous membrane

- Keep cool or use other preservation to prevent hatching and no need of preservation if needed for larva recovery

\section{Skin scrapings:}

- Should be sent dry and unpreserved in a plastic bottle.

- Scrape deep at the periphery of the lesion until blood oozes.

- If Ringworm is suspected, pull hair from edge of lesion with forceps and add to sample.

\section{Skin biopsy:}

- Cut about $1 \mathrm{~cm}$ square and deep enough to reach subcutaneous tissue. 
- Preserve in $10 \%$ buffered formalin for histopathology

- Use ice for skin biopsies to be screened for viruses e.g., LSD and Orf.

\section{Urine:}

- Should be collected in clean and sterile bottles for bacteriological and hormone residues examination.

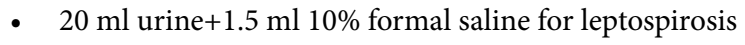

- $2.5 \%$ Boric acid can be used as a preservative if to be used for bacteriology

- Use faecal analysis bottles.

Pus:

- Swab aseptically and deep from the edge of the abscess.

- Put swab in commercial semi solid transport medium.

- Collect into a sterile syringe or test tube and keep refrigerated.

Fluids:

- This refers to fluids from joint, peritoneal, pleural, pericardial, cerebrospinal fluid, etc.

- Collect asceptically using a sterile needle and syringe/ vacatainers.

- Should be preserved on ice.

Milk:

- Ensure no antibiotic treatment was given prior to sampling
- Wash the teats with water

- Wash your hands and disinfect with $70 \%$ alcohol

- Dry off teats and disinfect with $70 \%$ alcohol

- Expel the foremilk then milk into a sterile bottle held almost horizontally to avoid dirt particles falling inside

- Submit within a short time while fresh

- Keep cool on ice.

\section{References}

1. Ballard B, Rockett $\mathrm{J}$ (2009) Restraint and Handling for Veterinary Technician and Assistants. Delmar, Cengage Learning, USA.

2. Frandson RD, Wilke WL, Fails AD (2009) Anatomy and Physiology of Farm Animals. 7th edn. Wiley-Blackwell, USA.

3. Jackson P, Cockcroft $P$ (2002) Clinical Examination of Farm Animals. Blackwell Science, UK.

4. Kahn CM (2010) Merck Veterinary Manual. 10th edn. Whitehouse Station, Merck, NJ, USA.

5. Kelly WR (1974) Veterinary Clinical Diagnosis. 2nd edn. Bailliere Tindal \& Casell, London, UK.

6. Radostits OM, Gay CC, Hinchcliff KW, Constable PD (2007) Veterinary Medicine: A textbook of the diseases of cattle, sheep, pigs and goats, horses. 10th edn. St. Louis: Saunders (Elsevier).

7. Riviere JE, Papich MG (2001) Veterinary Pharmacology \& Therapeutics. 9th edn. Wiley-Blackwell, USA.

8. Chauhan RS, Agarwal DK (2008) Textbook of Veterinary, Clinical and Laboratory Diagnosis. 2nd edn. Jaypee Publishers, New Delhi, India. 